\title{
Kinematic and chemical evolution of early-type galaxies ${ }^{\star} \star \star$
}

\author{
B. L. Ziegler ${ }^{1}$, D. Thomas ${ }^{2}$, A. Böhm ${ }^{1}$, R. Bender ${ }^{2,3}$, A. Fritz ${ }^{1}$, and C. Maraston ${ }^{2}$ \\ 1 Universitätssternwarte Göttingen, Geismarlandstraße 11, 37083 Göttingen, Germany \\ e-mail: bziegler@uni-sw.gwdg.de \\ 2 Max-Planck-Institut für extraterrestrische Physik, Giessenbachstraße, 85748 Garching, Germany \\ 3 Universitätssternwarte München, Scheinerstraße 1, 81679 München, Germany
}

Received 2 December 2004 / Accepted 7 December 2004

\begin{abstract}
We investigate in detail 13 early-type field galaxies with $0.2<z<0.7$ drawn from the Fors Deep Field. Since the majority ( 9 galaxies) is at $z \approx 0.4$, we compare the field galaxies to 22 members of three rich clusters with $z=0.37$ to explore possible variations caused by environmental effects. We exploit VLT/FORS spectra $(R \approx 1200)$ and HST/ACS imaging to determine internal kinematics, structures and stellar population parameters. From the Faber-Jackson and Fundamental Plane scaling relations we deduce a modest luminosity evolution in the $B$-band of $0.3-0.5$ mag for both samples. We compare measured Lick absorption line strengths ( $\mathrm{H} \delta, \mathrm{H} \gamma, \mathrm{H} \beta, \mathrm{Mg}_{b}$, and $\left.\mathrm{Fe} 5335\right)$ with evolutionary stellar population models to derive light-averaged ages, metallicities and the element abundance ratios $\mathrm{Mg} / \mathrm{Fe}$. We find that these three stellar parameters of the distant galaxies obey a scaling with velocity dispersion (mass) which is consistent with that of local nearby galaxies. In particular, the distribution of $\mathrm{Mg} / \mathrm{Fe}$ ratios of local galaxies is matched by the distant ones, and their derived mean offset in age corresponds to the average lookback time. This indicates that there was little chemical enrichment and no significant star formation within the last $\sim 5$ Gyr. The calculated luminosity evolution of a simple stellar population model for the derived galaxy ages and lookback times is in most cases consistent with the mild brightening measured by the scaling relations.
\end{abstract}

Key words. galaxies: elliptical and lenticular, $\mathrm{cD}$ - galaxies: evolution - galaxies: abundances - galaxies: stellar content galaxies: kinematics and dynamics - galaxies: distances and redshifts

\section{Introduction}

Recently, it has become feasible to study physical parameters of galaxies out to redshifts $z \approx 1$. Spectroscopy at $10 \mathrm{~m}$-class telescopes allows us to determine the internal kinematics such as rotational speed and velocity dispersion as well as the age and metallicity distribution of the stellar populations. Spatially high-resolution imaging with the Hubble Space Telescope (HST) reveals morphologies, characteristic sizes and total luminosities of distant galaxies. Particularly powerful are scaling relations that combine the kinematics (sampling both dark and bright matter) and the stellar populations (baryons). For dynamically hot galaxies, such scaling relations are the Faber-Jackson relation (FJR; Faber \& Jackson 1976) between the velocity dispersion $\sigma$ and the absolute luminosity and the Fundamental Plane (FP; Djorgovski \& Davis 1987; Dressler et al. 1987) encompassing effective radius $R_{\mathrm{e}}$, effective

^ Based on observations with the European Southern Observatory Very Large Telescope (ESO-VLT), observing run IDs 65.O-0049, 66.A-0547 and 68.A-0013.

$\star \star$ Based in parts on observations (PID 9502) with the NASA/ESA Hubble Space Telescope, obtained at the Space Telescope Science Institute, which is operated by AURA, Inc., under NASA contract NAS 5-26555. surface brightness $\langle\mu\rangle_{\mathrm{e}}$, and $\sigma$. The chemical enrichment history of the galaxies can be examined by comparing absorption line strengths to evolutionary stellar population models.

With these observational quantitative measurements, it is now possible to perform robust tests of galaxy formation theories. The basic prediction of cold dark matter (CDM) models of hierarchically growing structure is the gradual increase of mass of galaxies. Connecting this mass assembly with the physics of star formation leads to further predictions for the evolution of the stellar populations of a galaxy. The combination of characteristic velocities and sizes could in principle constrain the mass of galaxies, but this transformation is degenerate. The main problem is the cutoff radius for the dark matter halo which must follow certain assumptions by considering various constraints which greatly affect the estimated total mass of a galaxy. Thus, observations can better be taken to investigate the luminosity and chemical evolution and the age distributions of the stellar populations.

For spheroidal (elliptical) galaxies, CDM simulations yield a dependence on environment (e.g. Baugh et al. 1996). Galaxies born in high-density regions that evolve into rich clusters are cutoff from external influences much earlier on average than those in the field. Two thirds of the field ellipticals 
should still be produced at $z<1$ (e.g. Kauffmann et al. 1997), whereas the last major merger between two large galaxies in high density environments ending up in a big elliptical is predicted to occur at somewhat higher redshifts (e.g. Kauffmann 1996; Cole et al. 2000). These predictions manifest themselves in measureable parameters like mean age, metallicity and element abundances on the one side and a specific luminosity and color evolution on the other side. Thus, field ellipticals, in particular the bright ones, should on average have younger global ages (e.g. Kauffmann \& Charlot 1998) and more solarlike element abundance ratios (e.g. Thomas 1999) than cluster ellipticals.

The FJR \& FP scaling relations (e.g. Bender et al. 1992), the color-magnitude (e.g. Bower et al. 1992) and $\mathrm{Mg}-\sigma$ relations (e.g. Bender et al. 1993) of local early-type galaxies are very tight indicating that the bulk of their stars must have been formed at high redshifts $(z \gtrsim 2)$. Nevertheless, this homogeneity can be reconciled with the hierarchical merging scenario (Kauffmann 1996). Difficulties arise because of a probable degeneracy between age and metallicity effects, in particular for the $\mathrm{Mg}-\sigma$ relation (e.g. Trager et al. 2000; Kuntschner et al. 2002; Thomas et al. 2005).

But the Fundamental Plane is very well suited to study the redshift evolution of the luminosity (e.g. Bender et al. 1998) and the mass/light ratios (e.g. Franx 1993). Observations up to $z \approx 1$ were predominantly carried out for (bright and massive) galaxies in clusters. Here, consistent results were obtained of a very modest brightening and slow decrease of $M / L$ with $z$ fully compatible with the assumption of a very high formation redshift (e.g. van Dokkum et al. 1998; Kelson et al. 2000; van Dokkum \& Stanford 2003). On the other hand, findings for high $-z$ field galaxies are more discrepant. Some studies argue for a strong evolution (e.g. Treu et al. 2002; Gebhardt et al. 2003) whereas other authors favor a behaviour very similar to cluster galaxies (e.g. van Dokkum \& Ellis 2003; van der Wel et al. 2004). The differences might mainly come from the low number of observed galaxies. But the samples might also be affected by the so-called progenitor bias (van Dokkum \& Franx 2001) in the sense that the selection criteria for early-type galaxies differ strongly among researchers.

Many stellar population studies of the age and metallicity distribution have been carried out for local galaxies. The majority of cluster ellipticals have very old mean ages $(\approx 10 \mathrm{Gyr})$, high metallicities $\left(\approx 1-3 \times Z_{\odot}\right)$ and are enhanced in $\mathrm{Mg}$ over $\mathrm{Fe}$ compared to the solar ratio $([\mathrm{Mg} / \mathrm{Fe}] \approx 0.3)$ (e.g. Trager et al. 2000; Poggianti et al. 2001; Mehlert et al. 2003; Eisenstein et al. 2003; Thomas et al. 2005). There are trends between these parameters and the luminosity with less luminous galaxies having a wider spread and being skewed to younger ages. A more diverse behaviour is found for S0 galaxies in clusters. At least two families can be distinguished with one being very similar to the ellipticals and the other having younger luminosityweighted ages $(\approx 5 \mathrm{Gyr})$ indicating recent star formation activity. For field early-type galaxies, Kuntschner et al. (2002) find that they are younger (by 2-3Gyr) but more metal-rich (by $\approx 0.2 \mathrm{dex}$ ) than cluster galaxies exhibiting similar supersolar $\mathrm{Mg} / \mathrm{Fe}$ ratios. In their recent study of field and cluster galaxies compiling high-quality data from several sources,
Thomas et al. (2005) show that mean ages, metallicities, and $\alpha /$ Fe ratios correlate with galaxy mass $(\sigma)$. Both zero-point and slope of the $\alpha / \mathrm{Fe}-\sigma$ relation are independent of the environmental density, while the ages of objects in low density environments appear systematically lower accompanied by slightly higher metallicities.

Little work has been done yet investigating ages and metallicities from absorption lines in distant galaxies because the high $S / N$ needed for such an analysis is achieved only with long exposures even with large telescopes and because individual lines can be severely corrupted by terrestrial spectral features in the red wavelength regime. But models of galaxy formation can be constrained much more effectively by measurements at higher redshifts because differences among the predictions are much larger. Jones et al. (2000), for example, use combinations of $\mathrm{H} \delta$ with metal lines measured in co-added spectra of several E and S0 galaxies in clusters at $z=0,0.3,0.5$, respectively. They claim that there is no significant difference in the age-metallicity distribution between these two galaxy types at any redshift. Kelson et al. (2001) presented a study of the evolution of the $\mathrm{H} \gamma$ and $\mathrm{H} \delta$ Balmer absorption in galaxies in four clusters up to $z=0.8$. Assuming that the same relation between these indices and velocity dispersion holds at all redshifts, they derived a modest evolution in the zeropoint as expected for a passive evolution of old stellar populations and consistent with the evolution of $M / L$ determined from an FP analysis. Recently, Eisenstein et al. (2003) examined 22000 bright early-type galaxies from the SDSS averaging the spectra within bins of luminosity, environment, and redshift $(0<z<0.5)$ to produce high- $S / N$ mean spectra. They confirmed that these $L_{*}$ galaxies have mainly old quiescent stellar populations with high metallicities and an excess of $\alpha$ - (Mg-) elements with respect to the solar value independent of environment or redshift.

In this paper, we analyse the properties of galaxies at redshifts $z \approx 0.4$ both in clusters and in the field. In Sect. 2 our observations and data analysis is described. The Faber-Jackson relation and the Fundamental Plane are used to investigate the evolution in luminosity in Sect. 3. Measured absorption line strengths (Sect. 5) are compared in Sect. 6 to stellar population models that explicitely take into account variations in the abundance of elements to explore the chemical enrichment histories of the galaxies. A summary and discussion is presented in Sect. 7.

Throughout the paper, we adopt the "concordance" cosmology with $\Omega_{\text {matter }}=0.3, \Omega_{\lambda}=0.7$, and $H_{0}=70 \mathrm{~km} \mathrm{~s}^{-1} \mathrm{Mpc}^{-1}$ (e.g. Tonry et al. 2003). This yields a distance luminosity of 104.75 for the Coma cluster which is used as a local reference sample.

\section{Observations and data analysis}

\subsection{Elliptical galaxies from the Fors Deep Field}

The spectroscopic observations of our sample of field ellipticals were performed in parallel to those of late-type galaxies in the Fors Deep Field that form the basis of our analysis of 
the evolution of the Tully-Fisher relation (Ziegler et al. 2002; Böhm et al. 2004). The main differences are that the early-type galaxy candidates were placed onto slitlets independent of their position angles and were observed with more than one MOS setup in many cases to increase the respective exposure time. All in all, nine different setups with the FORS1\&2 instruments at the VLT were acquired in three different observing runs with a total integration time of $2.5 \mathrm{~h}$ for each setup. Typically, each setup contained 2-4 elliptical candidates.

Object selection was based on the deep UBgRI photometry of the Fors Deep Field (FDF), which is described e.g. in Heidt et al. (2003). Candidates were chosen according to their spectrophotometric type and their extended structureless appearance. Main criterium was the apparent brightness $(R \leq 22.0)$ in order to ensure sufficient $S / N$ in the absorption lines for a robust determination of line strengths and velocity dispersions. Photometric redshifts were restricted to $z_{\mathrm{p}}<0.6$ of objects with an early model SED type (for a description of the photometric redshift performance see e.g. Bender et al. 2001). Although the two-dimensional distribution of FDF objects indicated a cluster of galaxies with $z_{\mathrm{p}} \approx 0.33$ and our primary goal was to target field ellipticals, we did not preselect against such candidates. After the spectral analysis, we could confirm that 15 out of 32 observed different elliptical galaxy candidates are most probably members of a cluster. Taking our measurements of the radial velocities of these galaxies, the lower limit for the velocity dispersion of the cluster is $\sigma_{\mathrm{c}} \gtrsim 430 \mathrm{~km} \mathrm{~s}^{-1}$. Since the cluster center was not observed but only the outer parts, the true $\sigma_{\mathrm{c}}$ is probably larger. At $z=0.33$, the $\mathrm{Mg} 5170$ absorption feature is unfortunately corrupted due to terrestrial absorption (the $B$ band) making it impossible to accurately determine the internal velocity dispersion of a galaxy (see below). For that reason we do not include these cluster galaxies in our analysis and, from hence forward, when we speak of FDF galaxies only the field ellipticals are meant.

The elliptical candidates were spread out among the spiral candidates for each setup placing them on a region of the CCD corresponding to observed wavelengths where either the $\operatorname{Mg}_{b}$-feature $\left(\lambda_{0} \approx 5170 \AA\right)$ or the $G$-band $\left(\lambda_{0} \approx 4300 \AA\right)$ should have been visible according to the photometric redshifts. As is described in more detail in Böhm et al. (2004) the MOS spectroscopy was performed using grism 600R with order separation filter GG435 with FORS2 (September and October 2000) and FORS1 (October 2001) at the VLT. With slit widths of $1^{\prime \prime}$, a spectral resolution of $R \approx 1200$ was achieved, the spatial scale was $0.2^{\prime \prime} / \mathrm{pixel}$, and seeing conditions were sufficient (varying between $0.4^{\prime \prime}$ and $0.9^{\prime \prime} F W H M$ ) to meet the Nyquist theorem.

Image reduction followed the usual steps of bias subtraction, flat fielding, sky subtraction and wavelength calibration. Because some spectra exhibited spatial distortions, the images of each individual slitlet were extracted from the full frame after bias subtraction allowing the typical two-dimensional image reduction of long-slit spectroscopy. All images of one respective slitlet including the science, flatfield and arclamp frames were rectified in exactly the same manner with the procedure described in Jäger et al. (2004) to ensure correct wavelength calibration. The spectral rows of an object were averaged using a Horne-based (Horne 1986) algorithm as implemented in the image analysis software MIDAS ${ }^{1}$ (see e.g. Ziegler et al. 2001). Finally, the one-dimensional spectra of the respective exposures were summed. In those cases where a galaxy was observed in two or three different MOS setups, the wavelength ranges covered did not match each other exactly leading to varying final count rates at different wavelengths.

The internal velocity dispersions $\sigma$ and the radial velocities $v$ of the galaxies were determined applying the Fourier Correlation Quotient (FCQ) method with an updated version of Bender (1990). For each galaxy, $\sigma$ and $v$ were measured using 3-4 different kinematic standard stars. Deviations from star to star ranged from 2 to $20 \mathrm{~km} \mathrm{~s}^{-1}$. Since only one good template star (SAO 162947) was available that was observed with FORS like the galaxies, three more K giant stars (SAO 32042, SAO 80333 \& SAO 98087) were used which had been observed with MOSCA at the $3.5 \mathrm{~m}$-telescope at the Calar Alto observatory ${ }^{2}$ and have sufficiently resolved spectra $\left(\sigma_{*} \approx 55 \mathrm{~km} \mathrm{~s}^{-1}\right.$ around $\mathrm{Mg}_{b}$ ). FCQ was run on either a "red" part of a galaxy spectrum (ideally covering $\mathrm{H} \beta-\mathrm{Fe} 5335$, but sometimes only $\mathrm{Mg}_{b}$ or only $\mathrm{H} \beta$ could solely be used) or a "blue" part (around the $G$-band), in some cases (5 out of 13) in both regimes. In order to determine the instrumental resolution of the galaxy spectra, the widths of 3-5 emission lines of the respective arc spectrum used for the wavelength calibration were measured at wavelengths corresponding to $\mathrm{Mg}_{b}$ and the $G$-band separately. Typical instrumental resolutions are $\sigma_{i} \approx 90-100 \mathrm{~km} \mathrm{~s}^{-1}$. To provide the "correct" template for the respective instrumental broadening, each stellar spectrum was artificially smeared out to $\sigma_{i}$ before its usage in FCQ. In the Appendix, we tabulate the error-weighted averages of $\sigma$ and $v$ (corrected for the radial velocities of the template stars) and their respective errors for $13 \mathrm{FDF}$ field galaxies that had sufficient $S / N$. We give only either the values derived from the blue or the red spectral part depending on their quality, since only these values are used for the analysis below. The signal-to-noise of a galaxy's continuum was determined with FCQ, too, which was calibrated with Monte-Carlo simulations using the stellar spectra.

Absorption line strengths as defined in the Lick system (Worthey \& Ottaviani 1997; Trager et al. 1998) were measured as described in Ziegler et al. (2001). To this purpose, the galaxy spectra had been artificially broadened first to match the instrumental resolution of the stellar Lick/IDS spectra $\left(\sigma_{\text {IDS }} \approx\right.$ $210 \mathrm{~km} \mathrm{~s}^{-1}$ around $\mathrm{Mg}_{b}$ ) before the equivalent widths were calculated taking the redshifts into account. The measured values were then corrected for the decrease in strength caused by the broadening due to the galaxies' internal velocity dispersions. The correction functions for the different absorption lines were determined by simulating this effect with the stellar spectra. Lick indices of the galaxies can be badly affected if either the central bandpass or those that sample the pseudo-continuum

\footnotetext{
${ }^{1}$ ESO-MIDAS (Munich Image Data Analysis System) is developed and maintained by the European Southern Observatory.

2 The German-Spanish Astronomical Center (DSAZ or CAHA), operated by the Max-Planck-Institut für Astronomie, Heidelberg, jointly with the Spanish National Commission for Astronomy.
} 
of a line are redshifted into the region where telluric emission lines are so strong that the residual spectrum after sky subtraction is too noisy. A line gets totally corrupted if it falls onto the telluric absorption features of the $A$ - and $B$-band. Since the FDF galaxies have varying redshifts different lines are affected in each case. Therefore, the quality of each measurement was checked individually and we give quality flags along with the line strengths and their (statistical) errors in the Appendix. The presented values are still not directly comparable to the central values of local galaxies that were measured on the Lick system. The reason for this is that the distant galaxies are apparently so small that the slitlet of width $1^{\prime \prime}$ covered a large fraction of the light distribution, typically $1-2$ half-light radii $R_{\mathrm{e}}$. The observed line strengths are, therefore, only luminosityweighted average values, which are not equivalent to the central values if a radial gradient across the galaxy exists. To take this effect into account, the measured values were corrected based on the logarithmic gradients as they were determined by Mehlert et al. (2003) with high- $S / N$ spectra of Coma cluster galaxies (see also Jørgensen et al. 1995). The aperture of the 13 FDF galaxies were determined individually considering both the slitwidth and the number of rows that were averaged by the Horne extraction algorithm. The same correction procedure was applied to the velocity dispersions, too.

Structural parameters as well as the total brightness of a galaxy were measured on our HST/ACS images of the FDF. With a mosaic of four pointings, almost the entire field of the FDF was imaged through the $F 814 W$ filter by the ACS/WFC with a total exposure time of $2360 \mathrm{~s}$ per quadrant. Analysis was performed on the pipeline-reduced images with an additional cosmic ray rejection procedure. The two-dimensional surface brightness distribution of a galaxy was fitted using the GALFIT package of Peng et al. (2002) to determine total light and half-light radius. Four different fitting functions (pure de Vaucouleurs, pure Sersic with variable exponent $(n=1-4)$, and these two profiles in combination with an exponential disk component) were applied. Comparing the residual images and reduced $\chi^{2}$-quality values, the best fit to the observed light distribution of a galaxy was assessed, which was in most cases the combination of a Sersic plus disk component. To test the robustness of the profile analysis, 2D-surface brightness fits were also performed using the GIM2D package of Simard et al. (2002). Total magnitudes determined with the two methods agree well within the errors as well as with those values derived with the SExtractor package (Bertin \& Arnouts 1996) applied on the groundbased $I$ band images. The magnitudes were calibrated onto the Vega system with a zeropoint of 25.478 and a color term of $0.042(V-I)+0.012(V-I)^{2}$ (M. Sirianni, ESA STScI, priv. com.). Aperture colors ( $2^{\prime \prime}$ diameter) were taken from our groundbased photometry. K-corrections were calculated by convolving respective filter transmission curves with the redshifted SED of the elliptical template galaxy from Kinney et al. (1996). We checked for internal consistency by transforming observed $F 814 \mathrm{~W}$-magnitudes into rest-frame Johnson- $B$ as well as observed Fors filter magnitudes that best match the redshifted $B$-band ( $g$ for $0.2<z<0.3$ and $R$ for $0.3<z<0.7)$. The derived $B_{\text {rst }}$ values agree well with each other (differences are smaller than errors).
Structural parameters, total magnitudes as well as the distance luminosities for the cosmology we use are given in the Appendix.

\subsection{Elliptical galaxies in distant clusters}

To explore possible differences in galaxy evolution due to environment, we investigate here in addition to the field galaxies also early-type galaxies in clusters. Since the majority of the FDF galaxies have redshifts around $z \approx 0.4$, we take galaxies from three clusters at $z \approx 0.37$ : Abell 370, CL 0949+4409, MS 1512.4+3647. The image reduction and data analysis was already published in Ziegler \& Bender (1997); Bender et al. (1998); Saglia et al. (2000). In contrast to Ziegler \& Bender (1997), we here aperture correct line indices and the velocity dispersions using the logarithmic gradients to be fully consistent with the field ellipticals. All other corrections and the derivation of the Lick line indices were performed as described above. Half-light radii and total luminosities were determined on HST/WFPC2 images using the method described by Saglia et al. (1997). Combining de Vaucouleurs and exponential surface brightness profile fits with their procedure is equivalent to the GALFIT measurements used for the FDF galaxies, so that the field and cluster samples can be directly compared to each other here. Absolute magnitudes and pysical sizes were re-calculated for the cosmology adopted here.

\section{The kinematic scaling relations}

\subsection{The Faber-Jackson relation}

As the first of the scaling relations that combine the kinematics (representing both luminous and dark mass) with the stellar populations (baryons only) of galaxies we investigate the Faber-Jackson relation (FJR, Faber \& Jackson 1976). Here, the absolute $B$ magnitude and the velocity dispersion $\sigma$ of local cluster galaxies follow a tight relation. In Fig. 1, we compare the distant galaxies to the local sample of ellipticals in the Virgo and Coma clusters from Dressler et al. (1987). The straight line is a principal component fit to the nearby galaxies (Ziegler \& Bender 1997). For this comparison, we use the luminosities of the distant galaxies as derived from the ground-based photometry in order to have a somewhat larger sample (trends are the same when restricting to space-based magnitudes). The FDF data points match the distribution of the distant cluster galaxies very well. Both distant samples are tight and clearly offset to larger luminosities with respect to the local FJR. At low $\sigma$ a few galaxies with rather large offsets do exist in both samples. Excluding these outliers, the 17 distant cluster galaxies are brighter on average for given $\sigma$ by $\left\langle\Delta M_{B}^{\mathfrak{c}}\right\rangle=-0.55$ with a standard deviation of 0.25 . This general brightening is consistent with expectations from passive evolution models if a formation redshift of $z_{\mathrm{f}}=4$ is assumed. For a simple stellar population the predicted $B$-band evolution of such an object between $z=0$ (age 12 Gyr) and $z=0.4$ (age $8 \mathrm{Gyr}$ ) is $\Delta M_{B}^{\mathrm{m}}=-0.50$ (using models by Maraston (2005) for two times solar metallicity and Salpeter initial mass function). A similar offset is displayed by the ten FDF ellipticals: $\left\langle\Delta M_{B}^{\mathrm{f}}\right\rangle=-0.37$ with standard deviation of 0.27 . 


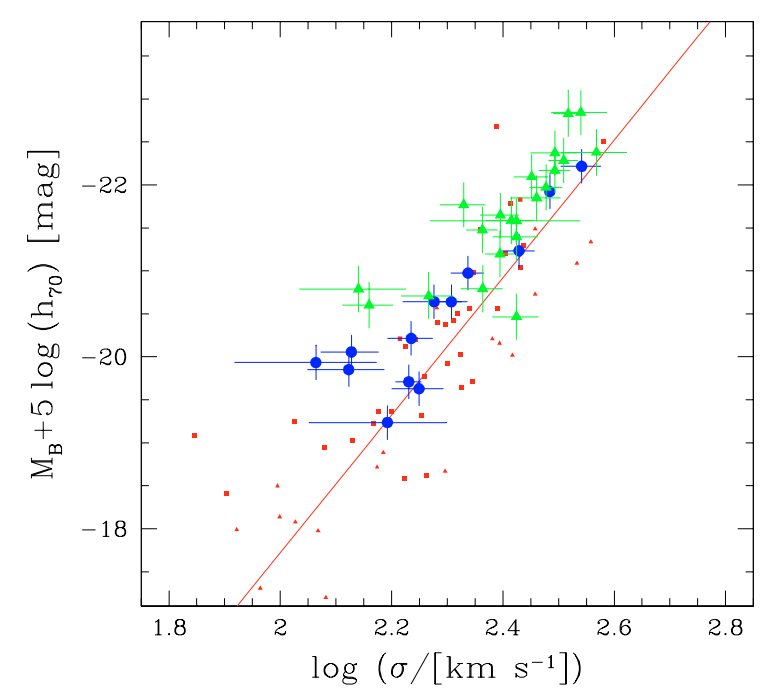

Fig. 1. Faber-Jackson relation between the central velocity dispersion and the absolute restframe $B$ magnitude. Local early-type galaxies in the Virgo (small triangles) and Coma (small squares) clusters are from Dressler et al. (1987), the straight line indicates their principal components fit (Ziegler \& Bender 1997). Both, ellipticals in distant clusters (large triangles) and in the field (large circles) follow a similar distribution, which is offset to larger luminosities.

\subsection{The Fundamental Plane relation}

In Fig. 2, we present the Fundamental Plane (FP) in the restframe $B$ band. The edge-on projection is chosen in a way that the distant-dependent parameter $R_{\mathrm{e}}$ (effective or half-light radius) is plotted on the $x$-axis, whereas the distant-independent parameters $\sigma$ and $\langle S B\rangle_{\mathrm{e}}$ (effective surface brightness) are displayed along the $y$-axis. The local sample which defines the FP comprises both elliptical and S0 galaxies in the Coma cluster from Dressler et al. (1987). Total luminosities and half-light radii of these galaxies were determined by Saglia et al. (1993) in exactly the same manner as our distant cluster galaxies by a simultaneous de Vaucouleurs and exponential fit to the respective surface brightness profile. The straight line indicates the principal components fit from Bender et al. (1998).

The distant galaxy samples displayed here are reduced by two (FDF) and six (clusters) objects with respect to the FJR analysis, because these galaxies are not visible on the respective HST images. Not counting the galaxy with the smallest and largest radius, the distant cluster members are on average brighter by $\left\langle\Delta M_{B}^{\mathfrak{c}}\right\rangle=-0.50$ with standard deviation of 0.19 . This assumes that the slope of the FP does not change within the $\sim 4 \mathrm{Gyr}$ lookback-time. This is equivalent to assuming that the structural parameters $R_{\mathrm{e}}$ and $\sigma$ do not change within that time and that only the luminosity of the stellar populations evolves. The derived value of the global luminosity increase is then again compatible with a pure passive evolution of the bulk of the stars that have already old ages $(\sim 8 \mathrm{Gyr})$ at the time of observations.

The sample of distant field galaxies lacks the large ellipticals and $\mathrm{cD}$ galaxies present in clusters of galaxies. On the contrary, the field sample encompasses some smaller galaxies. These objects display the largest luminosity offsets from the

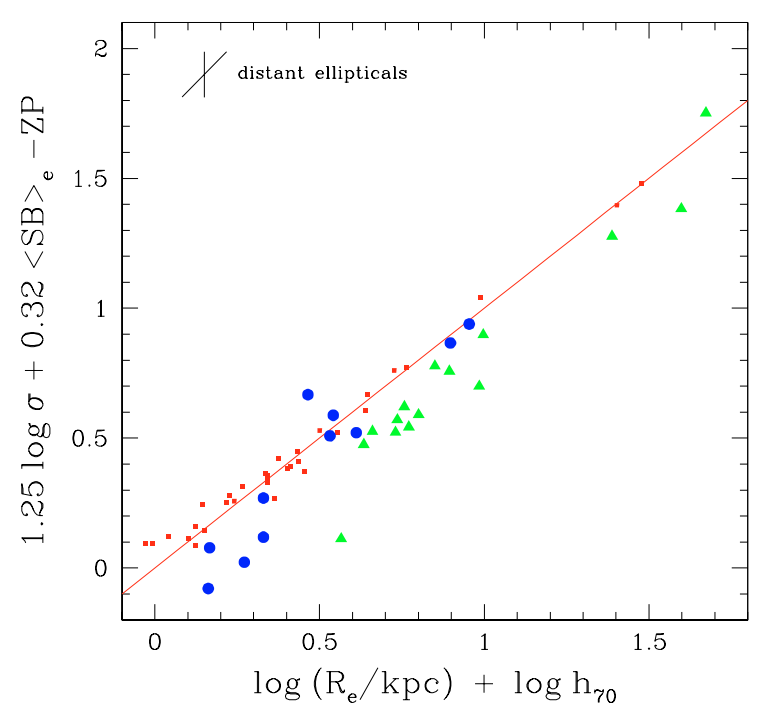

Fig. 2. The Fundamental Plane in $B$ seen edge-on. The local relation is a principal components fit to elliptical and S0 galaxies in Coma. Ellipticals in distant clusters are indicated by large triangles, distant field ellipticals by large circles. A typical errorbar is displayed in the upper left corner.

local FP, but lie still within $2 \sigma$ from the mean offset of the cluster galaxies. Excluding the two galaxies with "positive" evolution, the average brightening of the remaining nine FDF galaxies is $\left\langle\Delta M_{B}^{\mathrm{f}}\right\rangle=-0.34$ with standard deviation of 0.28 .

The one outlying galaxy (object FDF 6336) has a very elongated and distorted appearance on the HST image. Its very low Sersic index may indicate the presence of a disk but the disk/bulge ratio is very small. In the Kormendy diagram (relating $R_{\mathrm{e}}$ to $S B_{\mathrm{e}}$ ) it is also an outlier with either too faint a surface brightness or too small a half-light radius. The same behaviour is exhibited by object FDF 7796, which may have an overestimated $\sigma$. The combined effect makes this galaxy look "normal" in the FP. But its appearance is again very elongated and distorted on the ACS image and it may be associated with a companion galaxy. The galaxies which are likely to be lenticular galaxies judging from their appearance do follow the same distribution in the FP as the ellipticals without any prominent disk.

\section{The $\mathrm{Mg}_{b}-\sigma$ relation}

Another scaling relation that combines the kinematics to the stellar population is the $\mathrm{Mg}_{b}-\sigma$ relation (Bender et al. 1993). In the following sections and figures we compare the distant galaxies to the recent compilation of 124 early-type local galaxies by Thomas et al. (2005) that contains both morphological classes $\mathrm{E}$ and $\mathrm{S} 0$ and encompasses different environments from the field to clusters. They combined galaxies with high $S / N$ measurements of absorption line strengths and velocity dispersions selected from González-González (1993); Mehlert et al. (2003); Beuing et al. (2002) and Mendes de Oliveira et al. (2005). This compilation has the advantage for us that very similar methods to our procedures were used for the derivation of indices and $\sigma$ utilizing the same correction functions. Furthermore, model parameters of the stellar populations (see 


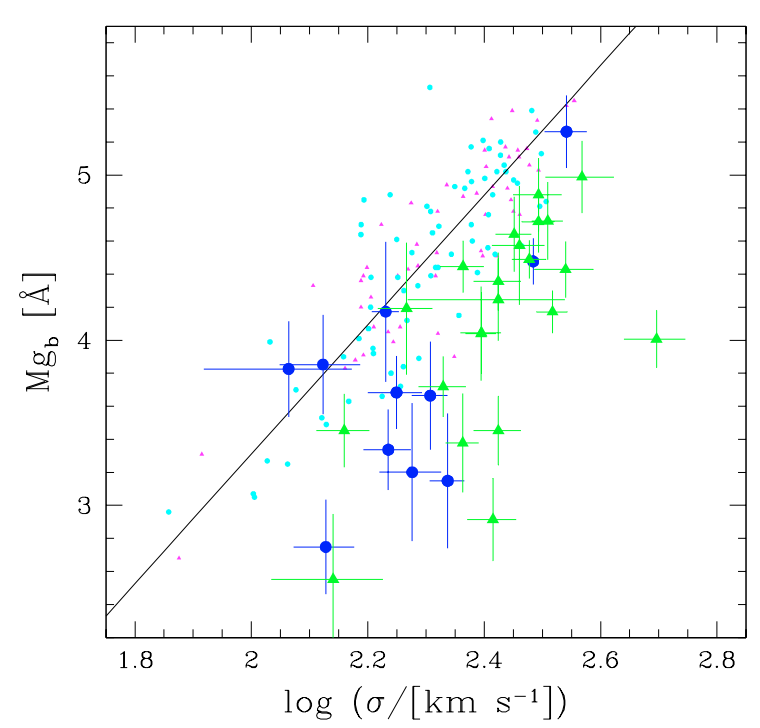

Fig. 3. $\mathrm{Mg}_{b}-\sigma$ relation between the central velocity dispersion and the central $\mathrm{Mg}_{b}$ line strength. Local galaxies (small symbols) are drawn from the compilation of Thomas et al. (2005) with E and S0 galaxies from the field (cyan circles) and clusters (magenta triangles). Both, ellipticals in distant clusters (green large triangles) and in the field (blue large circles) follow a similar distribution that is offset from the local relation (solid line) towards lower $\mathrm{Mg}_{b}$-strengths for given $\sigma$.

Sect. 6) are based on the same code. Thus, possible systematic biases between the distant and local samples are greatly reduced.

In Fig. 3, we show the $\mathrm{Mg}_{b}-\sigma$ relation of the local sample with the distant galaxies overplotted. We linearily fit the total local sample by a bisector, since differences between the various local subpopulations are significantly smaller than the average offset displayed by both distant samples. For fixed $\sigma, \mathrm{Mg}_{b}$ values are smaller with a mean offset of $-0.8 \AA$ in both cases. This can be interpreted as evolution in age and metallicity (Bender et al. 1996). To further explore this, we will compare below the measured equivalent widths of absorption features to evolutionary models of stellar populations in so-called line diagnostic diagrams (Sect. 5). In Sect. 6, we derive model ages and metallicities and explore their dependence on mass $\sigma$.

For galaxies with $\sigma>250 \mathrm{~km} \mathrm{~s}^{-1}$, the relation of the distant samples is as tight as for the local comparison galaxies. Smaller galaxies display a larger scatter which is compatible with the result by Thomas et al. (2005) that low-mass galaxies have more extended star formation histories. Ziegler \& Bender (1997) speculated whether some of the cluster galaxies with low $\mathrm{Mg}_{b}$ for their $\sigma$ might be post-starburst $\mathrm{E}+\mathrm{A}$ galaxies, where a recent $(\sim 1.5 \mathrm{Gyr}$ ago $)$ starburst diluted the $\mathrm{Mg}_{b}$ feature. Those $\mathrm{E}+\mathrm{A}$ galaxies are thought to be intermediate between spirals and ellipticals in the sense that two spirals are transformed into an early-type galaxy through merging. Although some local representatives of this galaxy type still have discernible disks, their surface brightness profiles are already bulge dominated (Yang et al. 2004) and their kinematics pressure supported (Norton et al. 2001). One of the characteristics of $\mathrm{E}+\mathrm{A}$ galaxies is their relatively strong absorption in the higher Balmer lines like $\mathrm{H} \delta$ compared to quiescent ellipticals. Unfortunately, we cannot address this issue for the cluster galaxies, because our spectra do not cover this "blue" part. Also, the [O III] 5007 line, if present in emission often used as indicator for ongoing star formation or for correcting the $\mathrm{H} \beta$ absorption for possible emission fill-in, falls into the atmospheric $B$ band at redshifts around 0.4 and, therefore, cannot be used for this investigation.

In the case of the FDF galaxies, we could measure $\mathrm{H} \delta$ for seven objects. But only three (FDF 1161, 4285, 7459) of the four FDF galaxies (\& FDF 6439) with seemingly too low an $\mathrm{Mg}_{b}$ for their $\sigma$ have $\mathrm{H} \delta$ measurements. The other four FDF objects with known $\mathrm{H} \delta$ (FDF 5011, 6307, 6336, 7796) are distributed in the $\mathrm{Mg}_{b}-\sigma$ plane like the other ones, although one of them (FDF 7796) has rather strong H $\delta$. Only one of the low$\mathrm{Mg}_{b}$ objects (FDF 7459) has very strong $\mathrm{H} \delta$ absorption which would be in compliance with a post-starburst model.

\section{Line diagnostic diagrams}

Absorption line strengths can be taken as measurements of the average age and metallicity of a stellar population. Within the Lick/IDS system, $\mathrm{H} \beta$ usually is taken as an indicator of age, whereas a combination of $\mathrm{Mg}_{b}, \mathrm{Fe} 5270$, and $\mathrm{Fe} 5335$ yields information on the mean metallicity $[\mathrm{Z} / \mathrm{H}]$ (e.g. Faber et al. 1985; Trager et al. 1998). Since a galaxy's spectrum is averaged over the slit aperture, any possible radial gradient of the lines is smeared out and the measurements represent luminosityweighted mean values only. The first effect is counterbalanced by correcting the observed line strength (see Sect. 2) according to aperture size assuming the validity of the locally determined radial gradients at higher redshifts. The age and metallicity of a stellar population can be deduced by comparing different indices in so-called line diagnostic diagrams to a model grid. Minimizing the differences between observed and model values, best fitting values for age and metallicity can be determined. Before we investigate the fitted model parameters in Sect. 6, we show the actual data in Figs. 4 and 5. As mentioned before, important absorption lines of a galaxy can be redshifted into the regime of strong terrestrial emission and absorption features, so that it is not possible to measure them with sufficient accuracy. For our distant galaxies at $z \approx 0.4$ one or more of the four traditional indices are affected. In order not to greatly reduce the samples, we do not combine Fe 5270 and $\mathrm{Fe} 5335$ to a mean $\mathrm{Fe}$ index $\langle\mathrm{Fe}\rangle$ nor do we add $\mathrm{Mg}_{b}$ to form $[\mathrm{MgFe}]$.

To investigate qualitatively age and metallicity effects, we plot in Fig. 4 only $\mathrm{H} \beta$ versus $\mathrm{Mg}_{b}$ with a model grid from Thomas et al. (2003) that takes into account the enhancement of $\mathrm{Mg}$ over $\mathrm{Fe}$ observed in most elliptical galaxies by fixing $[\mathrm{Mg} / \mathrm{Fe}]$ to 0.3 . The local cluster galaxies are mainly located in a region between solar and thrice solar $[\mathrm{Z} / \mathrm{H}]$ and old ages of 5-15 Gyrs (Thomas et al. 2005). The error-weighted mean values for the local cluster galaxies are $1.76 \AA$ for $\mathrm{H} \beta$ and $4.67 \AA$ for $\mathrm{Mg}_{b}$. The distant cluster ellipticals show a wide spread in $\mathrm{Mg}_{b}$ with accordingly sub- to supersolar metallicities. The error-weighted mean values are $1.74 \AA$ for $\mathrm{H} \beta$ and $4.28 \AA$ for $\mathrm{Mg}_{b}$. The statistics of the distant field ellipticals suffers from the low number of eight galaxies. Three galaxies have 


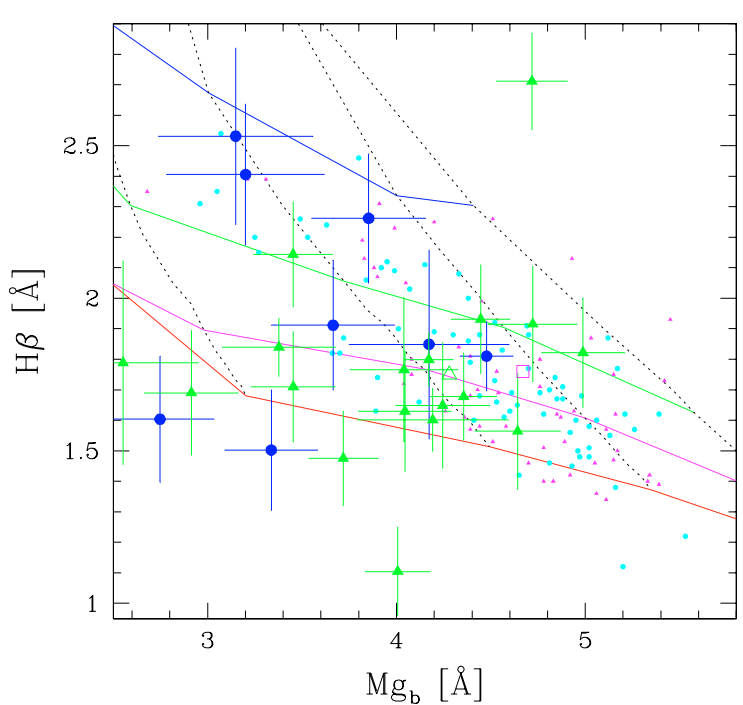

Fig. 4. The same samples (and symbols) as in Fig. 3, displaying $\mathrm{H} \beta$ vs. $\mathrm{Mg}_{b}$ taken as indicative of age and metallicity $[\mathrm{Z} / \mathrm{H}]$, respectively. Big open triangle and square denote the mean value of the distant and local cluster sample respectively. The lines connect model values from Thomas et al. (2003) enhanced in $\mathrm{Mg}$ so that $[\mathrm{Mg} / \mathrm{Fe}]=0.3$. Solid lines correspond to ages of 2, 5, 10,15 Gyr top to bottom and dashed lines to $[\mathrm{Z} / \mathrm{H}]=-0.33,0,0.33,0.67$ left to right.

intermediate populations similar to the mean of the distant cluster sample. Two FDF galaxies have such low $\mathrm{H} \beta$ that they fall below the "oldest" model line (15 Gyr) and seem to have subsolar $[\mathrm{Z} / \mathrm{H}]$, whereas three other ones are compatible with ages of 2-3 Gyrs with $1-2 \times$ solar $[\mathrm{Z} / \mathrm{H}]$ assuming simple stellar populations (SSPs).

To look for any possible enhancement of $\mathrm{Mg}$ over Fe with respect to the solar abundance ratio $[\mathrm{Mg} / \mathrm{Fe}]=0$, we plot in Fig. 5 the $\mathrm{Fe} 5335$ index versus $\mathrm{Mg}_{b}$. Local galaxies are located between $1-3 \times$ solar $[\mathrm{Mg} / \mathrm{Fe}]$. The distant objects scatter within the same range independent from being in a cluster or field environment.

\section{The stellar populations}

We now discuss the model parameters for the galaxies. For each individual galaxy best fitting model parameters were determined by a minimization algorithm as explained in detail in Thomas et al. (2005). The measured ( $\sigma$ and aperture corrected) line strengths were compared iteratively to predicted model line strengths where the $[\mathrm{Mg} / \mathrm{Fe}]$ ratio, metallicity $[\mathrm{Z} / \mathrm{H}]$, and age was varied. Observed strengths of $\mathrm{Mg}_{b}$ and Fe 5335 were used in combination whenever their measurement was trustworthy. These lines were simultanously fitted together with one of the Balmer indices $\mathrm{H} \beta, \mathrm{H} \gamma_{\mathrm{F}}$, or $\mathrm{H} \delta_{\mathrm{F}}$ (when available). With that procedure we took into account the dependence of these lines on the $\mathrm{Mg} / \mathrm{Fe}$ ratio, which is in particular important for the higher order Balmer lines (Thomas et al. 2004).

In Fig. 6, we investigate the scaling relations of the resulting stellar parameters with velocity dispersion found for local early-type galaxies (Thomas et al. 2005). Ages, metallicities and $\alpha / \mathrm{Fe}$ ratios correlate with $\sigma$ and therefore stellar mass. For the comparison, we now only take the 86 galaxies from the

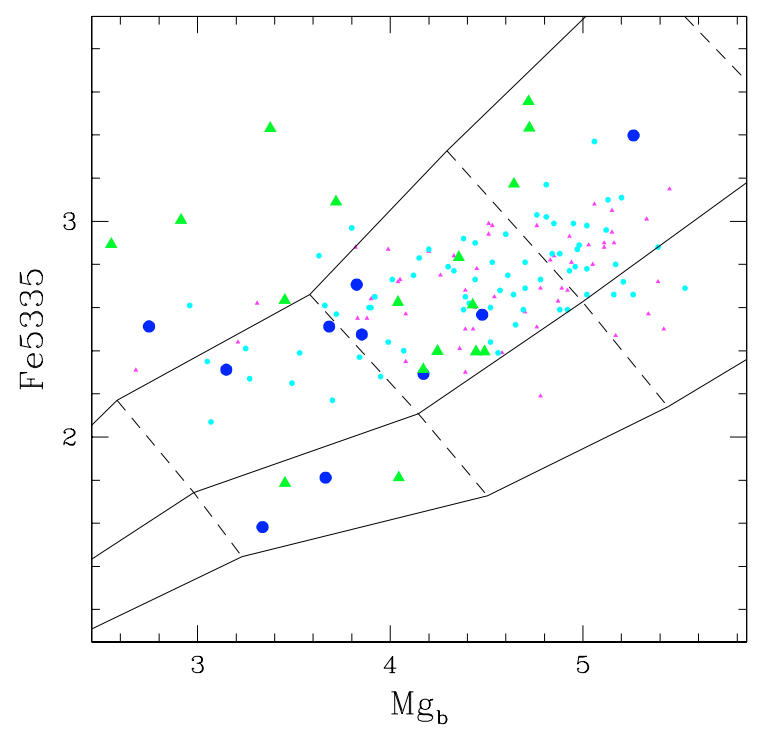

Fig. 5. The same samples (and symbols) as in Fig. 3, displaying $\mathrm{Fe} 5335$ vs. $\mathrm{Mg}_{b}$ to investigate the relative enrichment of $\mathrm{Fe}$ and $\mathrm{Mg}$. The lines connect model values from Thomas et al. (2003) for a constant age of $10 \mathrm{Gyr}$. Solid lines correspond to $[\mathrm{Mg} / \mathrm{Fe}]=0,0.3,0.5$ top to bottom and dashed lines to $[Z / H]=-0.33,0,0.33,0.67$ left to right.

local sample classified as being old ( $\gtrsim 5 \mathrm{Gyr}$ ), since the presented scaling relations were derived for this object class (Category 1) only. The local cluster and field samples differ slightly in the zeropoint of the linear fits for ages and for metallicities. In Fig. 6, we overplot the derived stellar population parameters of the distant galaxies. For this comparison, we add the respective look-back time to the age of a galaxy. For all three distributions, the distant samples nicely match the local ones. The individual data points scatter around the local fit lines, but not significantly larger than the local ones, and follow the same trends. In particular, the local $\alpha / \mathrm{Fe}-\sigma$ relation is also obeyed by the distant galaxies. This is in compliance with the derived ages that are younger only corresponding to their look-back times. From this we can conclude that for the majority of the local galaxies no significant star formation episodes and no further chemical enrichment has taken place within the last $\sim 5$ Gyr. Slight differences (which are not significant) in $[\mathrm{Z} / \mathrm{H}]-\sigma$ between the local and distant samples may be attributed to the fact, that the aperture correction in the case of the distant galaxies was done onto the nominal aperture whereas the local galaxies were measured within $1 / 10$ th of $R_{\mathrm{e}}$. Since age and $\alpha / \mathrm{Fe}$ have zero radial gradients in local galaxies (Mehlert et al. 2003), only our $[\mathrm{Z} / \mathrm{H}]$ determinations could be affected in the sense that they are systematically slightly too low.

\section{Summary and discussion}

We have investigated the kinematic, photometric, structural and stellar population parameters of a sample of field galaxies with mean redshift of 0.4 . The galaxies were drawn from the FORS Deep Field on the basis of their early-type SED, i.e. their optical and near-infrared colours. It turned out that they 


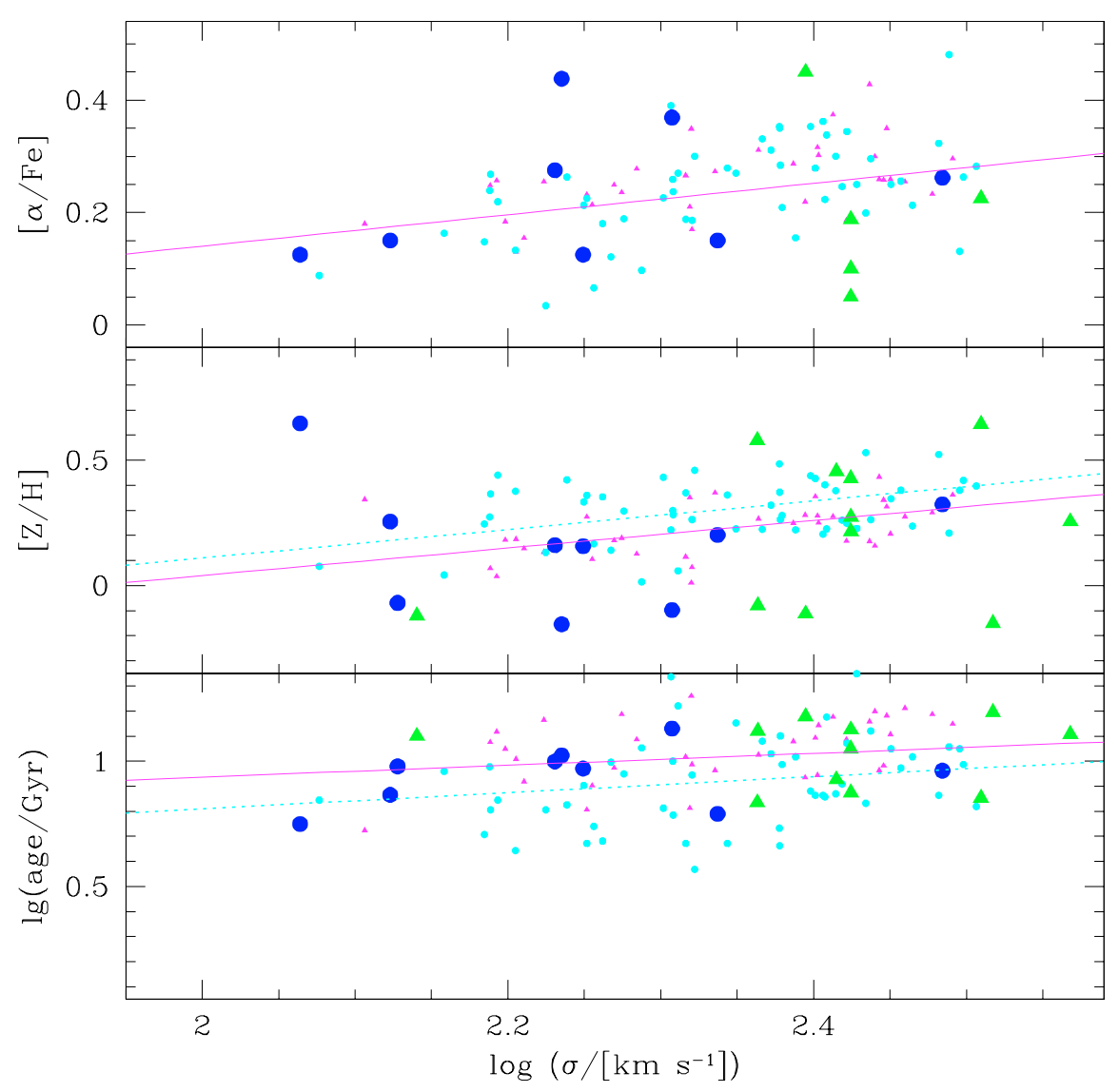

Fig. 6. The samples (and symbols) as in Fig. 3 except that the local sample is now restricted to Category 1 (ages older than $\sim 5$ Gyr) galaxies. The lines indicate the local relations between age, metallicity $[\mathrm{Z} / \mathrm{H}]$, and $[\alpha / \mathrm{Fe}]$ with velocity dispersion as derived through Monte Carlo simulations by Thomas et al. (2005), separately for the cluster (solid line) and field sample (dotted line). For the distant galaxies, the respective look-back times have been added to the derived ages.

all have indeed absorption-line spectra without any emission lines, i.e. without obvious indications of ongoing star formation. Their structure is mostly dominated by a bulge component, but a few galaxies clearly have disks resembling local lenticular S0 galaxies.

To learn about any possible evolution specific to the lowdensity environment, we compare the FDF sample to galaxies in three rich clusters at similar redshifts and to local samples both with field and cluster galaxies. We first explored the luminosity evolution via the Faber-Jackson and Fundamental Plane relations. Assuming that the structure (size) and mass (velocity dispersion) of elliptical galaxies do not change with time, we find consistently from both scaling relations a very modest evolution in brightness that can be modeled by passive evolution of a simple stellar population (SSP). The average increase of the $B$ luminosities by $0.3-0.5 \mathrm{mag}$ is found for both the distant field and cluster galaxies and can be explained by a high formation redshift $\left(z_{\mathrm{f}}>2\right)$ of their stars.

This result is consistent with those studies that also find a weak luminosity evolution and little difference between field and cluster ellipticals. In their FP study of 18 field early-type galaxies with $\langle z\rangle=0.4$, van Dokkum et al. (2001) also find that they are brighter in $B$ by 0.4 mag in general, which can be modeled with an SSP only slightly younger than in the case of their cluster galaxies. A similar conclusion was reached by
Rusin et al. (2003), who measured an increase by $0.5 \mathrm{mag}$ at $z=0.4$. In contrast, other groups like Treu et al. (2002) or Gebhardt et al. (2003) find that the evolution for field galaxies is stronger and compatible with a significantly younger mean age for their stellar populations than in the case of cluster galaxies. Restricting their samples to galaxies around $z=0.4$, $\Delta B$ amounts to $0.7-0.8 \mathrm{mag}$. The differences among the various studies become even more prominent at higher $z$, where the latter authors derive a much larger brightening for the field galaxies in comparison to cluster members. The rather small differences at $z \approx 0.4$ may arise from the still quite small numbers of observed galaxies per sample. These small samples may therefore also be more affected by the particular method to select the targets. Our FDF galaxies are, for example, redder by 0.7 mag on average in observed $(V-I)$ color than the Gebhardt et al. sample, which has $\langle(V-I)\rangle=1.3$ for 5 ellipticals at $0.3<z<0.5$, while both our field and cluster galaxies have $\langle(V-I)\rangle=2.0$. This indicates that our field galaxies probably predominantly trace the upper envelope of the age- $\sigma$ distribution.

Compatibility with SSP models of passive evolution is also found for the majority of the FDF galaxies in the $\mathrm{Mg}_{b}-\sigma$ relation. Their mean offset from the local relation is with $-0.8 \AA$ identical to the one for the distant cluster galaxies. The local slope is followed by the distant galaxies as well with a 
tendency for a slight steepening at the low-mass end. This indicates that age cannot be the principal driver of the main part of the relationship. Otherwise a significant steepening also at the high-mass end would be detected, as the $\mathrm{Mg}_{b}$ index of younger objects, that also have on average lower $\sigma$ according to the age- $\sigma$ correlation, would evolve faster to lower index values with increasing redshift. This result is in line with the conclusion drawn by several local studies (e.g. Trager et al. 2000; Kuntschner et al. 2002; Thomas et al. 2005) that the $\mathrm{Mg}_{b}-\sigma$ relation is driven by metallicity rather than by age.

To explore in more detail the distribution in metallicity, chemical enrichment and mean ages of the stellar systems, we compare diagnostic absorption lines from the Lick system to SSP models. To this purpose we simultaneously fit measured $\mathrm{Mg}, \mathrm{Fe}$, and Balmer indices by varying model ages, $[\mathrm{Z} / \mathrm{H}]$ metallicities, and $[\alpha / \mathrm{Fe}]$ ratios. We find a broad range in these model parameters for both distant samples, field and clusters. But their distributions are not random because all three stellar population parameters scale with velocity dispersion, and, therefore, to some degree with total mass, like their local counterparts.

A passive evolution of cluster galaxies from $z=0.5$ to 0 was also favoured by Jones et al. (2000) investigating the distribution of $\mathrm{H} \delta_{\mathrm{A}}$ with metal lines measured in co-added spectra. They detect no significant difference between morphologically classified S0 and E galaxies arguing also that the majority of S0 cluster members do not show any evidence of recent star formation activity as expected in some scenarios of galaxy transformation. Two of our FDF galaxies (FDF $6336 \&$ 7796) have extraordinary $\mathrm{H} \delta_{\mathrm{F}}$ absorption leading to young model ages under the assumption of a single stellar population. Their strengths may also be explained in a scenario where these galaxies had experienced a short intense star burst in their recent past involving only a small fraction of their mass. The ACS images reveal a significant disk component in these two galaxies, which may point to a very early spiral morphology. In that case a low-level continous star formation might be expected, but we do not detect any emission lines.

Kelson et al. (2001) investigated the higher-order Balmer lines $\mathrm{H} \delta_{\mathrm{A}}$ and $\mathrm{H} \gamma_{\mathrm{A}}$ of early-type galaxies in three clusters at $z=0.3,0.6$ and 0.8 claiming that there is a correlation with $\sigma$ with small scatter. Interpreting the offsets in the zeropoint for the three redshifts as caused by an evolution in the mean age of the stellar systems, they derive formation redshifts of $z_{\mathrm{f}} \gtrsim 2$ for SSP models assuming co-evality for all galaxies. Since we only have four FDF galaxies with high-quality measurements of the two indices (and none in the cluster sample), we cannot confirm or exclude a correlation with $\sigma$. But the assumption that all galaxies have roughly the same age must be rejected for our cluster galaxies.

We also investigated whether our findings derived from the Fundamental Plane and the absorption line diagnosis with respect to luminosity evolution are compatible with each other quantitatively. We determine the model brightening of our galaxies from the predicted restframe $B$ magnitudes for the respective ages and lookback times taking into account the derived $[\mathrm{Z} / \mathrm{H}]$ metallicities and $[\alpha / \mathrm{Fe}]$ ratios again using SSP models by Maraston (2005). In Fig. 7, we make this

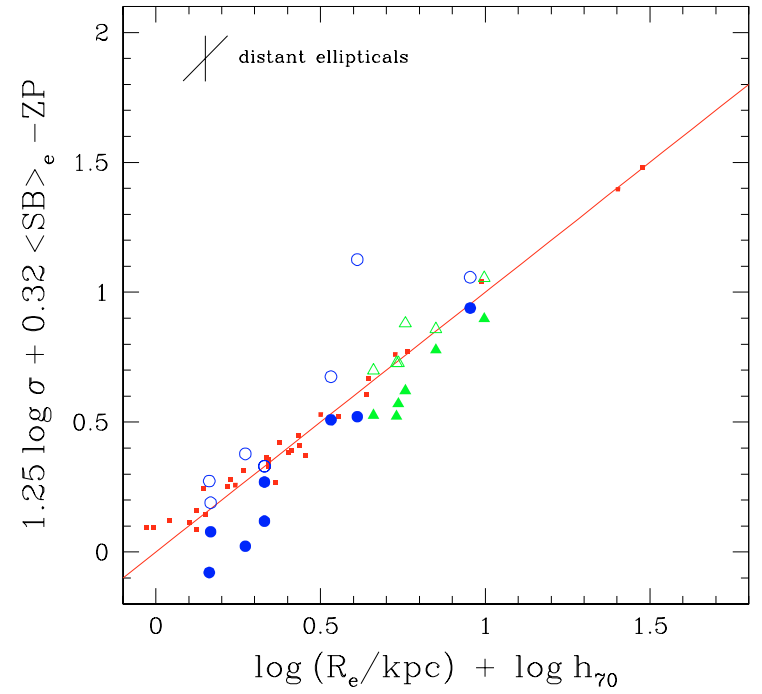

Fig. 7. The Fundamental Plane as in Fig. 2 but restricted to those galaxies for which we could derive light-averaged model ages of their stellar population. The open symbols represent the data "corrected" for the predicted brightening for each galaxy and nicely match the distribution of the local galaxies.

comparison by subtracting the predicted luminosity evolution from the observed data points. The evolution-corrected distant FP falls onto the local relation and nicely matches the distribution of the local galaxies. The one outlier is galaxy FDF 7796 for which we speculated above that it has undergone a recent starburst, thereby causing the light-averaged age to be very low.

In summary, we conclude from our study that the evolution of the stellar populations of the majority of early-type galaxies within the last $3-6 \mathrm{Gyr}$ is compatible with the scenario of passive evolution. Since the $\alpha / \mathrm{Fe}-\sigma$ and age- $\sigma$ relations have not changed since $z=0.4$, no significant star formation and chemical enrichment has occured since that time. To investigate certain issues in more detail, like any possible difference between low- and high-density environments, new studies with more objects are required with high $S / N$ measurements allowing the investigation of several aspects (FP \& line diagnostics) ideally out to higher redshifts. For this, we are currently analyzing another sample of distant absorption-line galaxies drawn from the William-Herschel Deep Field for which we also have VLT spectra and HST/ACS imaging with a similar quality as for the FDF galaxies presented here.

Acknowledgements. We thank the anonymous referee for a very quick response. We acknowledge fruitful discussions with all FDF consortium members, in particular with Dr. A. Gabasch, M. Pannella, Dr. R. P. Saglia, Dr. S. Seitz (all Munich \& Garching) and Dr. K. Jäger and B. Gerken (Göttingen). We thank J. Fliri and A. Riffeser (Sternwarte Munich) for applying their cosmic ray removal algorithm to the HST/ACS images. We are grateful to Drs. J. Heidt, D. Mehlert and S. Noll (Landessternwarte Heidelberg) for performing the FDF spectroscopic observations and thank ESO and the Paranal staff and the STScI and ST-ECF staff for efficient support. We also thank the PI of the FORS project, Prof. I. Appenzeller (Heidelberg) and Prof. K. J. Fricke (Göttingen) for providing guaranteed time for this project. This work has been supported by the Volkswagen Foundation (I/76520) and the BMBF/DLR (50 OR 0301) and made use of the ADS, astroph, and CDS data bases. 


\section{Appendix A: Parameters of the FORS Deep Field galaxies}

Table A.1. Basic parameters of the FORS Deep Field galaxies.

1. ID from Heidt et al. (2003), 2. redshift, 3. luminosity distance, 4. lookback time, 5. signal/noise, 6. velocity dispersion corrected for aperture (see Sect. 2), 7. its error, 8.-11. FORS total magnitudes (SExtractor's MAG_BEST), 12. absolute restframe $B$ magnitude, 3., 4., and 12. for $\Omega_{\text {matter }}=0.3, \Omega_{\lambda}=0.7, H_{0}=70 \mathrm{~km} \mathrm{~s}^{-1} \mathrm{Mpc}^{-1}$.

\begin{tabular}{cccccccccccc}
\hline \hline FDF & $z$ & $\mathrm{~d} l$ & $\begin{array}{c}t_{\mathrm{lb}} \\
{[\mathrm{Gyr}]}\end{array}$ & \multicolumn{1}{c}{$\begin{array}{c}\sigma \\
{\left[\mathrm{km} \mathrm{s}^{-1}\right]}\end{array}$} & $\begin{array}{r}\Delta \sigma \\
{\left[\mathrm{km} \mathrm{s}^{-1}\right]}\end{array}$ & $\begin{array}{c}B_{\mathrm{tot}} \\
{[\mathrm{mag}]}\end{array}$ & $\begin{array}{c}g_{\mathrm{tot}} \\
{[\mathrm{mag}]}\end{array}$ & $\begin{array}{c}R_{\mathrm{tot}} \\
{[\mathrm{mag}]}\end{array}$ & $\begin{array}{c}I_{\mathrm{tot}} \\
{[\mathrm{mag}]}\end{array}$ & $\begin{array}{c}M_{B} \\
{[\mathrm{mag}]}\end{array}$ \\
\hline 1161 & 0.40 & 2147 & 4.2 & 19 & 134 & 16 & 23.4 & 22.3 & 20.6 & 19.9 & -20.2 \\
4285 & 0.40 & 2145 & 4.2 & 29 & 172 & 16 & 23.2 & 22.1 & 20.4 & 19.6 & -20.4 \\
5011 & 0.65 & 3922 & 6.0 & 15 & 268 & 18 & 23.9 & 23.0 & 21.5 & 20.9 & -21.0 \\
5908 & 0.22 & 1112 & 2.7 & 127 & 305 & 5 & 19.7 & 18.4 & 17.1 & 16.5 & -21.9 \\
6307 & 0.45 & 2508 & 4.7 & 29 & 203 & 14 & 23.5 & 22.3 & 20.5 & 19.7 & -20.7 \\
6336 & 0.46 & 2561 & 4.7 & 8 & 156 & 43 & 24.7 & 23.7 & 21.9 & 21.1 & -19.4 \\
6338 & 0.41 & 2238 & 4.4 & 22 & 178 & 19 & 23.9 & $*$ & $*$ & 20.3 & -19.7 \\
6439 & 0.40 & 2145 & 4.2 & 41 & 217 & 15 & 22.4 & 21.4 & 19.7 & 18.9 & -21.0 \\
7116 & 0.46 & 2544 & 4.7 & 28 & 133 & 21 & 24.2 & 23.0 & 21.3 & 20.5 & -20.0 \\
7459 & 0.54 & 3100 & 5.3 & 20 & 189 & 23 & 24.3 & 22.9 & 21.2 & 20.3 & -20.6 \\
7796 & 0.41 & 2231 & 4.4 & 15 & 116 & 33 & 23.6 & 22.5 & 20.8 & 20.1 & -20.1 \\
8372 & 0.23 & 1142 & 2.7 & 57 & 170 & 9 & 21.6 & 20.7 & $*$ & 18.5 & -20.1 \\
8626 & 0.41 & 2226 & 4.4 & 27 & 348 & 29 & 21.4 & $*$ & $*$ & 17.7 & -22.4 \\
\hline
\end{tabular}

* No entry because object not visible in respective image.

Table A.2. Line strengths of fully corrected (see Sect. 2) Lick indices of the FORS Deep Field galaxies together with their errors and (by-eye) quality flags. Units are Å. Quality flags: 0: satisfactory, 1: a bit noisy, 2: in region of strong sky line, 6: affected by telluric $B$ band, 7: affected by telluric $A$ band, 8: affected by end of spectrum, 9: not visible.

\begin{tabular}{ccccccccccccc}
\hline \hline $\mathrm{FDF}$ & $\mathrm{H} \delta_{\mathrm{F}}$ & $\mathrm{dH} \delta_{\mathrm{F}}$ & $\mathrm{qH} \delta_{\mathrm{F}}$ & $\mathrm{H} \gamma_{\mathrm{F}}$ & $\mathrm{dH} \gamma_{\mathrm{F}}$ & $\mathrm{qH} \gamma_{\mathrm{F}}$ & $\mathrm{H} \beta$ & $\mathrm{dH} \beta$ & $\mathrm{qH} \beta$ & $\mathrm{Mg}_{b}$ & $\mathrm{dMg}_{b}$ & $\mathrm{qMg}_{b}$ \\
\hline 1161 & 1.3 & 0.2 & 0 & -0.6 & 0.2 & 0 & 1.6 & 0.2 & 0 & 2.7 & 0.3 & 1 \\
4285 & 1.2 & 0.2 & 0 & -0.7 & 0.2 & 0 & 1.5 & 0.2 & 0 & 3.3 & 0.2 & 0 \\
5011 & 1.0 & 0.2 & 0 & 0.4 & 0.2 & 0 & 1.9 & 0.3 & 1 & $*$ & $*$ & 9 \\
5908 & $*$ & $*$ & 9 & $*$ & $*$ & 9 & 1.8 & 0.1 & 0 & 4.5 & 0.1 & 0 \\
6307 & 0.8 & 0.2 & 2 & -0.8 & 0.3 & 2 & 1.9 & 0.2 & 0 & 3.7 & 0.3 & 0 \\
6336 & 2.6 & 0.3 & 0 & -0.7 & 0.3 & 2 & 0.5 & 0.3 & 2 & $*$ & $*$ & 7 \\
6338 & $*$ & $*$ & 9 & $*$ & $*$ & 8 & $*$ & $*$ & 6 & 3.7 & 0.2 & 0 \\
6439 & $*$ & $*$ & 9 & $*$ & $*$ & 8 & 2.5 & 0.3 & 0 & 3.1 & 0.4 & 0 \\
7116 & $*$ & $*$ & 9 & -0.4 & 0.2 & 2 & 2.3 & 0.2 & 0 & 3.9 & 0.3 & 1 \\
7459 & 2.5 & 0.2 & 2 & -0.1 & 0.2 & 0 & 2.4 & 0.2 & 0 & 3.2 & 0.4 & 1 \\
7796 & 2.0 & 0.2 & 0 & 1.0 & 0.2 & 0 & $*$ & $*$ & 6 & 3.8 & 0.3 & 0 \\
8372 & $*$ & $*$ & 9 & $*$ & $*$ & 8 & 1.8 & 0.3 & 0 & 4.2 & 0.4 & 2 \\
8626 & $*$ & $*$ & 9 & $*$ & $*$ & 8 & $*$ & $*$ & 6 & 5.3 & 0.2 & 0 \\
\hline
\end{tabular}


Table A.3. Fully corrected Fe 5335 Lick index and SSP model ages, metallicities $[\mathrm{Z} / \mathrm{H}]$, and $\mathrm{Mg} / \mathrm{Fe}$ ratios $[\alpha / \mathrm{Fe}]$ of the FORS Deep Field galaxies. Average errors in $\log (\mathrm{age}),[\mathrm{Z} / \mathrm{H}]$, and $[\alpha / \mathrm{Fe}]$ are $0.18 \mathrm{dex}, 0.2 \mathrm{dex}$, and $0.15 \mathrm{dex}$, respectively. In Col. 8 it is indicated, which Balmer line was used to derive the model age. The last four columns are based on the HST/ACS images: ACS total $I$ magnitude (GALFIT), mean surface brightness in $B$ within $R_{\mathrm{e}}$ corrected for cosmological dimming, and half-light radius (GALFIT). Physical units for $\Omega_{\text {matter }}=0.3, \Omega_{\lambda}=0.7$, $H_{0}=70 \mathrm{~km} \mathrm{~s}^{-1} \mathrm{Mpc}^{-1}$.

\begin{tabular}{|c|c|c|c|c|c|c|c|c|c|c|c|}
\hline FDF & $\begin{array}{c}\text { Fe } 5335 \\
\AA\end{array}$ & $\begin{array}{c}\mathrm{dFe} 5335 \\
\AA\end{array}$ & qFe 5335 & $\begin{array}{l}\text { age } \\
\text { Gyr }\end{array}$ & {$[\mathrm{Z} / \mathrm{H}]$} & {$[\alpha / \mathrm{Fe}]$} & $\begin{array}{l}\text { Balmer } \\
\text { line }\end{array}$ & $\begin{array}{c}I_{\text {tot }} \\
{[\mathrm{mag}]}\end{array}$ & $\begin{array}{c}\left\langle\mu_{B}\right\rangle_{\mathrm{e}} \\
\text { [mag/sq.as.] }\end{array}$ & $\begin{array}{c}R_{\mathrm{e}} \\
{[\operatorname{arcsec}]}\end{array}$ & $\begin{array}{l}\log R_{\mathrm{e}} \\
{[\mathrm{kpc}]}\end{array}$ \\
\hline 1161 & 2.5 & 0.3 & 1 & 5.3 & -0.07 & -0.09 & $\mathrm{H} \gamma_{\mathrm{F}}$ & 19.9 & 20.3 & 0.40 & 0.33 \\
\hline 4285 & 1.6 & 0.3 & 2 & 6.3 & -0.15 & 0.44 & $\mathrm{H} \delta_{\mathrm{F}}$ & 19.9 & 20.3 & 0.40 & 0.33 \\
\hline 5011 & $*$ & $*$ & 9 & $*$ & $*$ & $*$ & $*$ & 20.8 & 20.6 & 0.50 & 0.54 \\
\hline 5908 & 2.6 & 0.2 & 0 & 6.5 & 0.32 & 0.26 & $\mathrm{H} \beta$ & 16.3 & 21.5 & 2.50 & 0.95 \\
\hline 6307 & 1.8 & 0.5 & 0 & 8.8 & -0.10 & 0.37 & $\mathrm{H} \beta$ & 19.8 & 20.8 & 0.59 & 0.53 \\
\hline 6336 & $*$ & $*$ & 9 & $*$ & $*$ & $*$ & $*$ & 21.2 & 21.8 & 0.50 & 0.46 \\
\hline 6338 & 2.5 & 0.3 & 0 & 5.0 & 0.16 & 0.12 & $\mathrm{H} \beta$ & $*$ & $*$ & $*$ & $*$ \\
\hline 6439 & 2.3 & 0.5 & 0 & 1.9 & 0.20 & 0.15 & $\mathrm{H} \beta$ & 19.1 & 19.2 & 0.35 & 0.27 \\
\hline 7116 & 2.5 & 0.4 & 2 & 2.6 & 0.25 & 0.15 & $\mathrm{H} \beta$ & 20.6 & 19.7 & 0.25 & 0.16 \\
\hline 7459 & $*$ & $*$ & 9 & $*$ & $*$ & $*$ & $*$ & $*$ & $*$ & $*$ & $*$ \\
\hline 7796 & 2.7 & 0.4 & 0 & 1.3 & 0.65 & 0.12 & $\mathrm{H} \gamma_{\mathrm{F}}$ & 20.1 & 21.8 & 0.75 & 0.61 \\
\hline 8372 & 2.3 & 0.4 & 0 & 7.2 & 0.16 & 0.28 & $\mathrm{H} \beta$ & 18.6 & 19.8 & 0.40 & 0.17 \\
\hline 8626 & 3.4 & 0.2 & 1 & $*$ & $*$ & $*$ & $*$ & 17.9 & 21.0 & 1.45 & 0.90 \\
\hline
\end{tabular}

\section{References}

Baugh, C. M., Cole, S., \& Frenk, C. S. 1996, MNRAS, 283, 1361 Bender, R. 1990, A\&A, 229, 441

Bender, R., Appenzeller, I., Böhm, A., et al. 2001, in Deep Fields, ed. S. Christiani, A. Renzini, \& R. Williams, ESO Astrophysics Symposia (Berlin: Springer), 96

Bender, R., Burstein, D., \& Faber, S. M. 1992, ApJ, 399, 462

Bender, R., Burstein, D., \& Faber, S. M. 1993, ApJ, 411, 153

Bender, R., Saglia, R. P., Ziegler, B., et al. 1998, ApJ, 493, 529

Bender, R., Ziegler, B., \& Bruzual, G. 1996, ApJ, 463, L51

Bertin, E., \& Arnouts, S. 1996, A\&AS, 117, 393

Beuing, J., Bender, R., Mendes de Oliveira, C., Thomas, D., \& Maraston, C. 2002, A\&A, 395, 431

Böhm, A., Ziegler, B. L., Saglia, R. P., et al. 2004, A\&A, 420, 97

Bower, R., Lucey, J. R., \& Ellis, R. S. 1992, MNRAS, 254, 601

Bruzual, G. A., \& Charlot, S. 1993, ApJ, 405, 538

Cole, S., Lacey, C. G., Baugh, C. M., \& Frenk, C. S. 2000, MNRAS, 319, 168

Djorgovski, S., \& Davis, M. 1987, ApJ, 313, 59

Dressler, A., Lynden-Bell, D., Burstein, D., et al. 1987, ApJ, 313, 42

Eisenstein, D. J., Hogg, D. W., Fukugita, M., et al. 2003, ApJ, 585, 694

Faber, S. M., Friel, E. D., Burstein, D., \& Gaskell, C. M. 1985, ApJS, 57, 711

Faber, S. M., \& Jackson, R. E. 1976, ApJ, 204, 668

Franx, M. 1993, PASP, 105, 1058

Gebhardt, K., Faber, S. M., Koo, D. C., et al. 2003, ApJ, 597, 239

González-González, J. d. J. 1993, Ph.D. Thesis, University of California, Santa Cruz

Heidt, J., Appenzeller, I., Gabasch, A., et al. 2003, A\&A, 398, 49

Horne, K. 1986, PASP, 98, 609

Jäger, K., Ziegler, B. L., Böhm, A., et al. 2004, A\&A, 422, 941
Jones, L., Smail, I., \& Couch, W. J. 2000, ApJ, 528, 118

Jørgensen, I., Franx, M., \& Kjærgaard, P. 1995, MNRAS, 276, 1341

Kauffmann, G. 1996, MNRAS, 281, 487

Kauffmann, G., \& Charlot, S. 1998, MNRAS, 294, 705

Kauffmann, G., Charlot, S., \& White, S. D. M. 1997, MNRAS, 283, L117

Kelson, D. D., Illingworth, G. D., Franx, M., \& van Dokkum, P. G. 2001, ApJ, 552, L17

Kelson, D. D., Illingworth, G. D., van Dokkum, P. G., \& Franx, M. 2000, ApJ, 531, 184

Kinney, A. L., Calzetti, D., Bohlin, R. C., et al. 1996, ApJ, 467, 38

Kuntschner, H., Smith, R. J., Colless, M., et al. 2002, MNRAS, 337, 172

Maraston, C. 2005, MNRAS, submitted [arXiv:astro-ph/0410207]

Mehlert, D., Thomas, D., Saglia, R. P., Bender, R., \& Wegner, G. 2003, A\&A, 407, 423

Mendes de Oliveira, C., Ziegler, B. L., Thomas, D., Maraston, C., \& Bender, R. 2005, A\&A, submitted

Norton, S. A., Gebhardt, K., Zabludoff, A. I., \& Zaritsky, D. 2001, ApJ, 557, 150

Peng, C. Y., Ho, L. C., Impey, C. D., \& Rix, H. 2002, AJ, 124, 266

Poggianti, B. M., Bridges, T. J., Carter, D., et al. 2001, ApJ, 563, 118

Rusin, D., Kochanek, C. S., Falco, E. E., et al. 2003, ApJ, 587, 143

Saglia, R. P., Bender, R., \& Dressler, A. 1993, A\&A, 279, 75

Saglia, R. P., Bertschinger, E., Baggley, G., et al. 1997, ApJS, 109, 79

Saglia, R. P., Maraston, C., Greggio, L., Bender, R., \& Ziegler, B. 2000, A\&A, 360, 911

Simard, L., Willmer, C. N. A., Vogt, N. P., et al. 2002, ApJS, 142, 1

Thomas, D. 1999, MNRAS, 306, 655

Thomas, D., Maraston, C., \& Bender, R. 2003, MNRAS, 339, 897

Thomas, D., Maraston, C., Bender, R., \& Mendes de Oliveira, C. 2005, ApJ, accepted [arXiv:astro-ph/0410209] 
Thomas, D., Maraston, C., \& Korn, A. 2004, MNRAS, 351, L19

Tonry, J. L., Schmidt, B. P., Barris, B., et al. 2003, ApJ, 594, 1

Trager, S. C., Faber, S. M., Worthey, G., \& González, J. J. 2000, AJ, 120,165

Trager, S. C., Worthey, G., Faber, S. M., Burstein, D., \& Gonzalez, J. J. 1998, ApJS, 116, 1

Treu, T., Stiavelli, M., Casertano, S., Møller, P., \& Bertin, G. 2002, ApJ, 564, L13

van der Wel, A., Franx, M., van Dokkum, P. G., \& Rix, H.-W. 2004, ApJ, 601, L5

van Dokkum, P. G., \& Ellis, R. S. 2003, ApJ, 592, L53

van Dokkum, P. G., \& Franx, M. 2001, ApJ, 553, 90 van Dokkum, P. G., Franx, M., Kelson, D. D., \& Illingworth, G. D. 1998, ApJ, 504, L17

van Dokkum, P. G., Franx, M., Kelson, D. D., \& Illingworth, G. D. 2001, ApJ, 553, L39

van Dokkum, P. G., \& Stanford, S. A. 2003, ApJ, 585, 78

Worthey, G., \& Ottaviani, D. L. 1997, ApJS, 111, 377

Yang, Y., Zabludoff, A. I., Zaritsky, D., Lauer, T. R., \& Mihos, J. C. 2004, ApJ, 607, 258

Ziegler, B. L., \& Bender, R. 1997, MNRAS, 291, 527

Ziegler, B. L., Böhm, A., Fricke, K. J., et al. 2002, ApJ, 564, L69

Ziegler, B. L., Bower, R. G., Smail, I. R., Davies, R. L., \& Lee, D. 2001, MNRAS, 325, 1571 